\title{
Criticality of Bitcoin Market
}

\author{
F. ChudZYŃski ${ }^{a, *}$ AND Z. STRUZIK ${ }^{a, b}$ \\ ${ }^{a}$ University of Warsaw, \\ Krakowskie Przedmieście 26/28, 00-927 Warsaw, Poland \\ ${ }^{b}$ The University of Tokyo, \\ 7-3-1 Hongo, Bunkyo-ku, Tokyo 113-8656, Japan
}

Doi: 10.12693/APhysPolA.139.447

*e-mail: filipchudzynski@gmail.com

\begin{abstract}
In this paper, we present an analysis of the non-Gaussianity of the Bitcoin market, in which we focus on the scale dependence of the variance $\lambda^{2}$ estimated by fitting the Castaing equation to the detrended price series. Our analysis showed scale invariance across a large range of scales in the years 2012-2019,
\end{abstract} which indicates that the Bitcoin market is in a critical state.

topics: criticality, invariance, Bitcoin, non-gaussian

\section{Introduction}

The stock market is said to be a complex system, i.e., a system consisting of many subsystems interacting in a manner, which proves predicting its macroscopic features - based on its constituents difficult. One of the features of stock price or currency exchange dynamics is that it is impossible to choose a characteristic scale of analysis. Conversely to everyday experience in which average and normal distribution might work as a sufficient approximation to predict qualities of many encountered objects, price fluctuations of returns do not follow this expectation [1]. They possess fractal properties [2], which means that the time-series is selfsimilar across scales [3] and thus needs to be analyzed accordingly. What follows is that one might expect to find critical phenomena and phase transitions in the price index of financial assets.

Criticality and long-range correlation tend to manifest themselves via scale invariance observed in, e.g., different systems such as human heart rate [4]. It was suggested that critical phenomena or, in other words, the instability of the system in finances can be linked to market crashes [5]. Indeed, it was demonstrated that 2 min S\&P 500, which exhibits scaling law in variance $\lambda^{2}$, entered a critical state manifested with data collapse during the market crash in 1987 [6].

To investigate the link between criticality and market crash more closely we decided to examine the Bitcoin, which is known for its huge price swings and speculative character [7]. We were able to show scale invariance in the range of hours persisting from 2012 up to 2019, which suggests that Bitcoin might be in a critical state not only during the crash.

\section{Methodology}

In this work, we focus on the variance $\lambda$ of the Bitcoin price in the sense which will be underlined in this section. We start with the fact that the distribution of returns is non-Gaussian with a key property of a much larger probability of occurrence of rare events - big differences in price to the average value, which is commonly referred to as "long/fat tails" [5]. Following the methodology used for S\&P 500 by Kiyono et al. [6], we assume phenomenologically that increments can be modeled by the random multiplicative process with increment in the form of

$$
\Delta_{s} Z(t)=\xi_{s}(t) \mathrm{e}^{\omega_{s}(t)},
$$

where $\xi_{s}$ and $\omega_{s}$ are independent Gaussian random variables with zero mean and variance $\sigma_{s}^{2}$ and $\lambda_{s}^{2}$, respectively. The $\omega_{s}$ models long tails and $\xi_{s}$ are responsible for changing the sign of the increment. The standardized probability density function of $\Delta_{s} Z$ has the form of

$$
\begin{gathered}
P_{\lambda, A}(x)=\int_{0}^{\infty} \frac{\mathrm{d} \sigma}{\sigma} \frac{1}{\sqrt{2 \pi} \sigma} \exp \left(-\frac{x^{2}}{2 \sigma^{2}}\right) \\
\times \frac{A}{\sqrt{2 \pi} \lambda} \exp \left(-\frac{\ln ^{2}\left(\sigma+\lambda^{2}\right)}{2 \lambda^{2}}\right) .
\end{gathered}
$$

The non-standardized version of $P_{\lambda, A}$ is called the Castaing equation and is introduced to describe the log-normal cascade model in a turbulent flow [8]. Parameter $\lambda$ controls the long tails and "sharpness" of the peak of PDF (Fig. 1), whereas $A$ is introduced to fit non-normalized histograms. For $\lambda=0$, the Castaing equation reduces to a Gaussian function. 


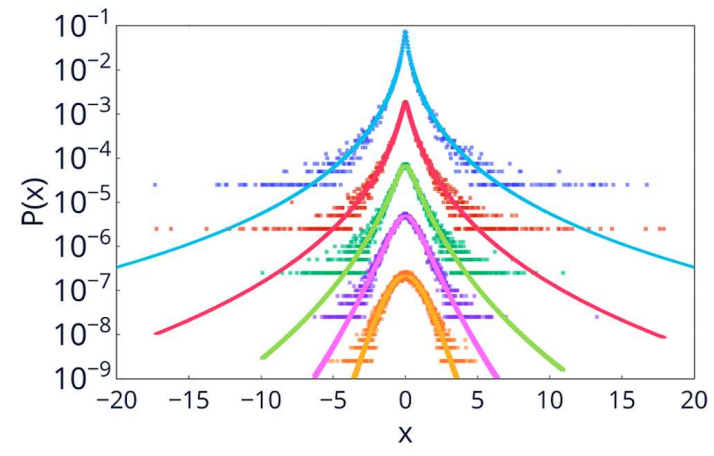

Fig. 1. The Castaing function fitted to a nonGaussian sequence plotted in semi-log scale. The non-Gaussian sequence was generated using (3), with $\lambda$ set to (from top to bottom) $1,0.8,0.6,0.4,0.2, N=40000$, bins $=12 \sqrt{N}$.

To estimate the probability density function from financial market data, we have removed the trend in windows from the logarithm of the price and then, for the detrended data, the log-returns were calculated. Standardized log-returns were used to estimate $P_{\lambda, A}$ and subsequently $\lambda$ from the histograms.

\subsection{Procedure of estimating $\lambda$}

Bitcoin one minute "close" price in USD $\{X(t)\}$ from Bitstamp exchange ${ }^{\dagger 1}$ is presented in Fig. 2. Data is non-stationary and prior to analysis needs to be detrended.

A full procedure is presented below.

1. NaNs (NaN - Not a Number) are filtered out from data.

2. Data is divided into batches - approximately one year, half year.

3. Detrending procedure:

- Logarithm of Bitcoin price $\{\log (X(t))\}$ is divided into intervals of length $2 s$ : $[1+s(k-1), s(k+1)]$, where $s$ is the window size and $k$ is the index of the window.

- In each subinterval, a linear function is fitted using linear regression ${ }^{\dagger 2}$. Deviation from the fitted function $\left\{X^{*}(t)\right\}$ is used to calculate detrended log-returns defined as: $\Delta_{s} Z(t)=X^{*}(t+s)-X^{*}(t)$.

\section{Histogram:}

- The standardization ${ }^{\dagger 3}$ of $\left\{\Delta_{s} Z(t)\right\}$ is performed for each scale $s$.

\footnotetext{
${ }^{\dagger 1}$ Downloaded from www.kaggle.com/gizemakbay/bitcoinhistorical-data-analysis.

${ }^{\dagger}$ The used functions come from Python library Scikit learn.

${ }^{\dagger}$ numpy
}

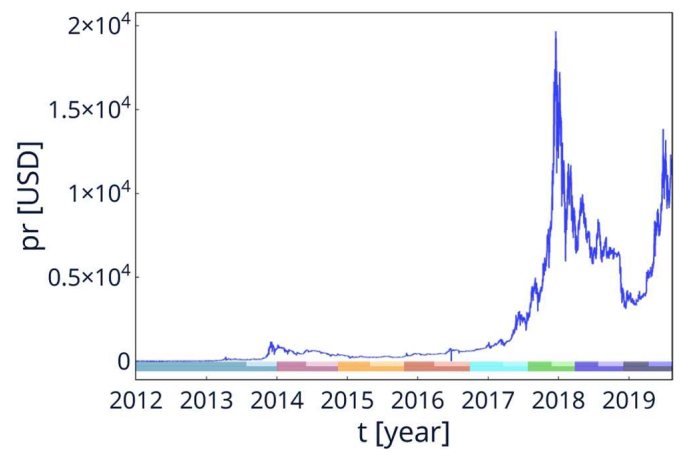

Fig. 2. Bitcoin prices between January 2012 and August 2019. Rectangles under the curve represent year and quarter-year batches. Colors correspond to the ones in Figs. 4 and 5. The 0th batch is much longer because of the missing data.

- The histogram is calculated ${ }^{\dagger 3}$ with the number of bins equal to the square root of length of the time series multiplied by 12 (this number was chosen based on simulation, see Sect. 3.4 for details).

\section{Fitting:}

- To the calculated histograms $P_{\lambda, A}$ is fitted using the least square method ${ }^{\dagger 4}$ with two parameters $\lambda$ and $A$.

\subsection{Activity periods}

Additionally to variance analysis, we have investigated activity on the Bitcoin market based on the volume transfer. In principle, there are no boundaries, when one can exchange Bitcoins, but one might expect that, e.g., working hours of normal stock exchanges might influence the trade of Bitcoins. To check activity periods, we measured how much volume was traded in specific periods $T$. This analysis showed in which time window most of the transaction occurs and allowed to identify the ana$\log$ of the trading day for the Bitcoin market.

\section{Results}

\subsection{Batches}

First, we need to comment on the 0th batch, which spans on a disproportionally larger time frame than the rest. This and all the other differences in sizes of the batches to time frame are a result of the fact that the batches are chosen based on the amount of non-NaN points, not based on the exact time frames. This also means that the unit of scales is only approximately in minutes.

${ }^{\dagger 4} \operatorname{lmfit}$ 


\subsection{Scale dependence of $\lambda$}

Results of the fitting procedure are presented using a histogram of detrended time-series from the 3rd batch. Based on the fit, we obtained the estimate of the variance $\lambda^{2}$ for each scale. First, we observe almost no change in the shape of the distributions and then a slow convergence to Gaussian distribution for $s>300$ (Fig. 3). Subsequently, we check other batches by investigating the estimated values of $\lambda^{2}$ vs. scale-dependence (Figs. 4 and 5) for both year and half-year batches. We find persistent scale invariance for most of the batches. It is especially easy to see how remarkably different in quality this result is, if we compare it with previous results for S\&P, based on which we would expect to see a power-law relation here, but instead we observe that Bitcoin has a characteristic scale. It is worth noting that also for $\mathrm{S} \& \mathrm{P}$, there seemed to be a small region of saturation below $s<20$, which might indicate that for less speculative markets than Bitcoin criticality also exists, but in much shorter scales [6].

Additionally, we observe this invariance for some of the curve clusters around different values of $\lambda^{2}$ (half-year batches $2,4,6,7$ for $\lambda^{2} \approx 0.25$ and $3,10,11,12$ for $\left.\lambda^{2} \approx 0.4\right)$.

Finally, we note that variance $\lambda^{2}$ is converging more slowly to Gaussian with respect to what was found for S\&P 500. Convergence below $\lambda^{2}<0.1$ did not occur for some of the batches for $s=8191$ in contrast to S\&P 500 for which the same convergence occurred for most of the batches at $s \approx 200$.

Observation of a consequent scale invariance indicates that the Bitcoin market is in highly correlated critical state in which there is a large probability of occurrence of rare events. Persistence of non-Gaussianity for such a large scale range made us investigate further what causes the correlation to diminish after $s>300$.

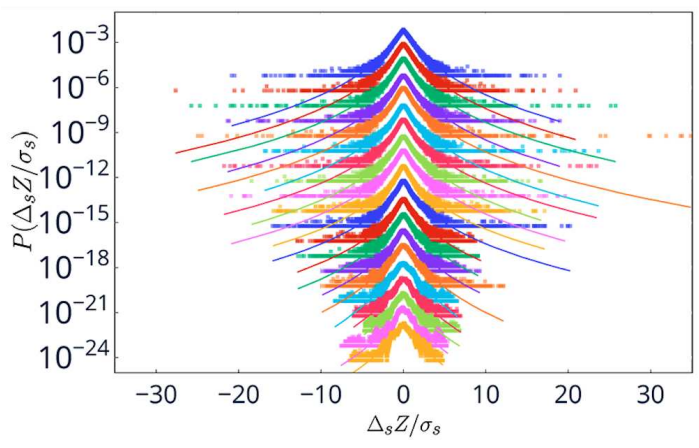

Fig. 3. The Castaing equation fitted to the 3rd yearly batch using the least square method to the histogram of $\Delta_{s} Z(t)$ for 20 points equidistant in a log scale range from 7 to 8192 (from top to bottom). Note that $\lambda^{2} \approx 0.44$ is independent of scale till $s=307$, after which it starts to converge to 0 .

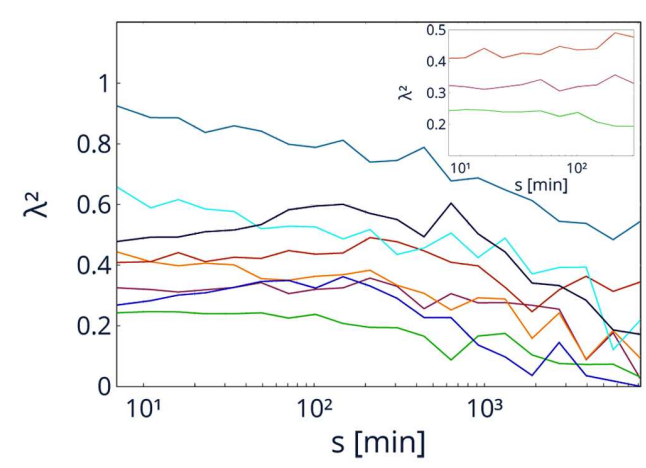

Fig. 4. Variance $\lambda^{2}$ as a function of scale estimated from yearly batches. For $s<300, \lambda^{2}$ is not converging to Gaussian and shows scale invariance with exception of the 0th and 4th batches, which show a gradual decrease. In the inset, the most prominent examples of invariance are shown.

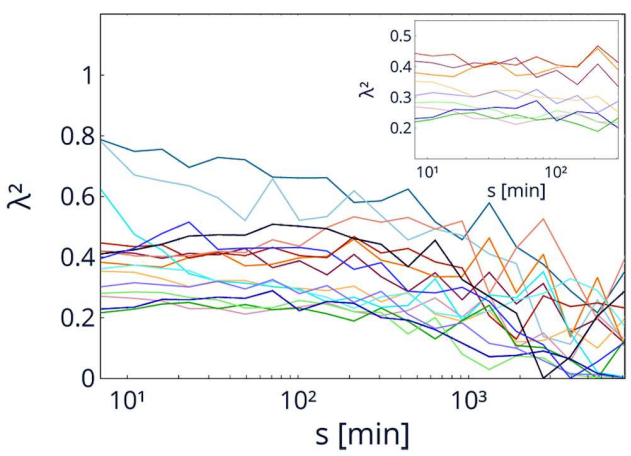

Fig. 5. Similarly as in Fig. 4, in half-year batches (with exception of the 0th, 1st, and 8th) we observe invariance of $\lambda^{2}$ to scale. Invariance occurs for different values of $\lambda^{2}$ between 0.2 and 0.5 .

\subsection{Volume}

Our analysis of volume exchange (Fig. 6) shows that in a $6 \mathrm{~h}$ interval $T$, more than $90 \%$ of volume has been traded (almost all for an $8 \mathrm{~h}$ interval). Periods in which most transactions occurred (Fig. 7) are 15-21 (15-23) UTC, which corresponds to 1117 (11-19) EST. However, $s=300$ corresponds to a $5 \mathrm{~h}$ period, not $6(8)$, but since the data consists of a large number of NaNs, it is difficult to estimate the exact time interval of scale invariance. Most importantly, we want to emphasize here that the scale invariance is linked to the activity on the market throughout the day and might be a hint that after $s=300$ the Bitcoin market is starting to selfregulate itself, which is manifested as a convergence toward Gaussian.

\subsection{Bin size optimization}

The analysis of variance in this paper relies on histograms. This measn that to get the best results, the number of bins should be optimized.

To do so, we generated a non-Gaussian time series sample with a given parameter $\lambda$, corresponding to the PDF $P_{\lambda, A}$ in (2) [9]:

$$
x_{i}=\xi_{i} \mathrm{e}^{\lambda \omega_{i}-\lambda^{2}}
$$




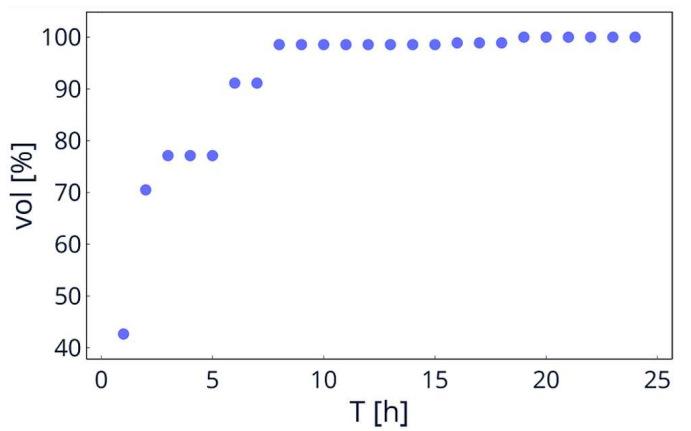

Fig. 6. Maximum volume traded in each time period $T$. Note that $90 \%$ of transactions occurs in $6 \mathrm{~h}$ period and almost $100 \%$ of transaction occurs in $8 \mathrm{~h}$ period.

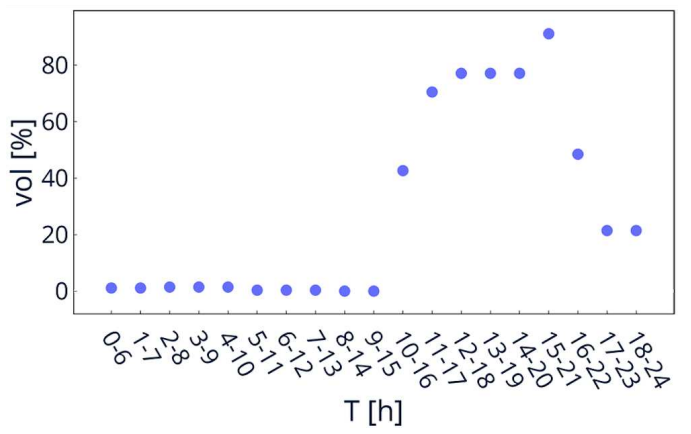

Fig. 7. Volume traded in each 6 -h period. Note that $90 \%$ of transactions occurred between 15 and 21 UTC.

where $\xi_{i}$ and $\omega_{i}$ are normal variables. Based on this time series, we fitted the Castaing equation and estimated parameter $\lambda$ (see Fig. 1).

From this procedure, we could quantify the influence of two parameters: sample size and number of bins on the average error $(|\lambda-\hat{\lambda}|)$ and spread of $\hat{\lambda}$ measured with standard deviation from 10 samples. We could then ask which parameters give us the demanded accuracy and precision, e.g., 0.1 and 0.1 , respectively. This is what is presented in Fig. 8, where $N$ stands for a sample size and $\sqrt{N} b$ is equal to the number of bins used to calculate the histogram. From the plot, we can see that there is an area between $N \in(20000,40000)$ and $b \in(8,15)$, which allows us to estimate $\lambda=1$ with accuracy and precision of 0.1 .

\section{Summary}

In this work, we were able to show that variance $\lambda^{2}$ is independent of scale for $s<300$ and that variance converges much slower to Gaussian for Bitcoin with respect to previously examined S\&P 500 . This indicates strong correlations and criticality in the market. Additionally, the analysis of volume showed that the duration of the period of strong correlations corresponds to the duration of the period in which most transactions take place during the day on the Bitcoin market.

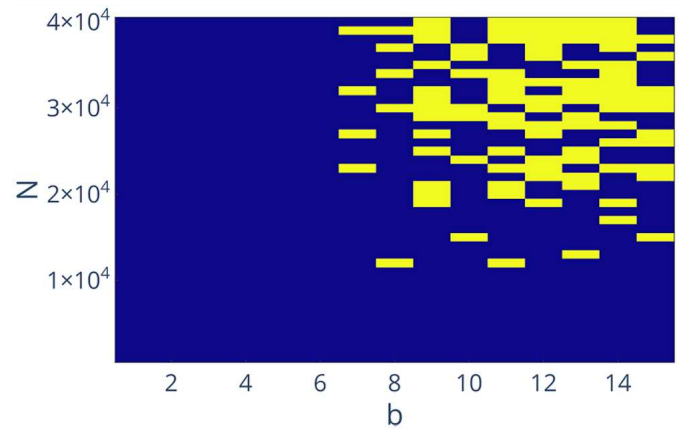

Fig. 8. Heatmap presents the combination of parameters, which allow (yellow) to estimate $\lambda=1$ with precision 0.1 and accuracy 0.1 .

The most important question is whether $\mathrm{S} \& \mathrm{P}$ from the years 1984-1995 is a reliable candidate for comparisons with Bitcoin. The issue worth further studying is how non-speculative markets are currently correlated. It might be the case that due to the use of the internet in trading and ease of communication, herding behavior and rare events became much more common.

We used Plotly [10] to plot our results. One can find the library we used to produce all the calculations in our online repository [11].

\section{References}

[1] E.E. Peters, Fractal Market Analysis Applying Chaos Theory to Investment and Economics, 1st ed., Wiley, 1994.

[2] P. Oświęcimka, S. Drożdż, J. Kwapień, A.Z. Górski, Acta Phys. Pol. A 117, 637 (2010).

[3] R. Hardstone, S.-S. Poil, G. Schiavone, R. Jansen, V.V. Nikulin, H.D. Mansvelder, K. Linkenkaer-Hansen, Frontiers Physiol. 3, 450 (2012).

[4] K. Kiyono, Z.R. Struzik, N. Aoyagi, S. Sakata, J. Hayano, Y. Yamamoto, Phys. Rev. Lett. 93, 178103 (2004).

[5] D Sornette, Phys. Rep. 378, 1 (2003).

[6] K. Kiyono, Z.R. Struzik, Y. Yamamoto, Phys. Rev. Lett. 96, 068701 (2006).

[7] D.G. Baur, KiHoon Hong, A.D. Lee, J. Int. Financ. Markets Institut. Money 54, 177 (2018).

[8] B. Castaing, Y. Gagne, E.J. Hopfinger, Physica D Nonlin. Phenom. 46, 177 (1990).

[9] K. Kiyono, Z. Struzik, Y. Yamamoto, Phys. Rev. E 76, 041113 (2007).

[10] Plotly Technologies Inc. Collaborative data science, 2015.

[11] Authors Repository. 